\title{
Comparison of the Change in the Prevalence and Intensity of Schistosoma haematobium Infection Between High and Low Prevalence Areas of White Nile State, Sudan
}

\author{
Seungman Cha ${ }^{1, \dagger}$, Sung-Tae Hong ${ }^{2, \dagger}$, Jin-Su Lee ${ }^{3}$, Hoo Gn Jeong ${ }^{3}$, In-Sun Kwon 4 , Abd Al Wahab Saed ${ }^{5}$, \\ Mousab Siddig Elhag ${ }^{6}$, Hassan Ahmed Hassan Ahmed Ismail', Mutamad Amin ${ }^{7}$, Young-Ha Lee, ${ }^{8, *}$ (C) \\ ${ }^{1}$ Graduate School of Global Development and Entrepreneurship, Handong Global University, Pohang 37554, Korea; ${ }^{2}$ Department of Tropical \\ Medicine and Parasitology, Seoul National University College of Medicine, Seoul 03080, Korea; ${ }^{3}$ Korea Association of Health Promotion, Seoul \\ 07653, Korea; ${ }^{4}$ Clinical Trial Center, Chungnam National University Hospital, Daejeon 35015, Korea; ${ }^{5}$ Ministry of Health, White Nile State, \\ Kosti, Sudan; ${ }^{6}$ Communicable and Non-Communicable Diseases Control Directorate, Federal Ministry of Health, Khartoum, Sudan; ${ }^{7}$ Research and \\ Grants Unit, Ahfad University for Women, Omdurman, Khartoum, Sudan; ${ }^{8}$ Department of Infection Biology and Department of Medical Science,
} Chungnam National University College of Medicine, Daejeon 35015, Korea

\begin{abstract}
This study aimed to investigate whether mass drug administration (MDA) intervention has an equivalent effect on reducing the prevalence and intensity of Schistosoma haematobium infection regardless of the baseline values. A repeated cross-sectional survey was performed targeting students of 12 primary schools in Al Jabalain and El Salam districts of White Nile State, Sudan, at both 1 week before and 8 months after the MDA. Prior to the baseline survey, schoolaged children in Al Jabalain had received MDA interventions twice in 4 years, while those in El Salam had not. The baseline prevalence was $9.1 \%$ in Al Jabalain and $35.2 \%$ in El Salam, which were reduced to $1.8 \%$ and $5.5 \%$ at 8 months after the MDA, respectively. The corresponding reduction rates were $80.3 \%$ and $84.4 \%$, not significant difference between both districts. However, changes in the geometric mean intensity (GMI) of egg counts were significantly different between both districts. The baseline GMls were 14.5 eggs per $10 \mathrm{ml}$ of urine (EP10) in Al Jabalain and 18.5 EP10 in El Salam, which were reduced to 7.1 and 11.2 EP10 after treatment, respectively. The corresponding reduction rates were $51.0 \%$ and $39.5 \%$. In conclusion, MDA interventions were found to bring about similar relative reduction in prevalence regardless of the baseline value; however, the relative reduction in infection intensity was more salient in the district with a low baseline value for both prevalence and intensity. This clearly points to the importance of repeated MDA interventions in endemic areas, which will eventually contribute to schistosomiasis elimination.
\end{abstract}

Key words: Schistosoma haematobium, prevalence, infection intensity, mass drug administration, Sudan

\section{INTRODUCTION}

Schistosomiasis is a parasitic disease caused by a blood fluke of the Schistosoma species. Humans are usually infected when they come into contact with contaminated freshwater and experience hematuria, anemia, enlargement of the liver and spleen, and growth retardation [1]. Schistosomiasis is endemic in 78 countries and is a major public health problem in many tropical countries-particularly in Africa, where $>90 \%$ of the global burden of schistosomiasis occurs [2]. The global bur-

- Received 14 June 2020, revised 27 July 2020, accepted 28 July 2020.

*Corresponding author (yhalee@cnu.ac.kr)

${ }^{\dagger}$ These authors contributed equally to this work.

(c) 2020, Korean Society for Parasitology and Tropical Medicine

This is an Open Access article distributed under the terms of the Creative Commons Attribution Non-Commercial License (https://creativecommons.org/licenses/by-nc/4.0) which permits unrestricted non-commercial use, distribution, and reproduction in any

medium, provided the original work is properly cited. den of schistosomiasis was conservatively estimated at 1.43 million disability-adjusted life years in 2017 [3]. More than 220.8 million people required preventive chemotherapy for schistosomiasis in 2017, and 102.3 million of them were treated [4]. Sudan has one of the highest schistosomiasis prevalence rates in the world [5-8]. About $15 \%$ of the total population ( $\sim 5.8$ million people) require treatment, with the majority being children [9].

Mass drug administration (MDA) refers to the delivery of preventive chemotherapy to targeted populations irrespective of individual infection status. Currently, 5 major "tool-ready" neglected tropical diseases with MDA are lymphatic filariasis, onchocerciasis, schistosomiasis, soil-transmitted helminthiasis, and trachoma [10]. MDA of praziquantel to at-risk populations has become the cornerstone of schistosomiasis control, especially for school-aged children (SACs), who have the high- 
est risk of infection $[11,12]$. So far, most studies on the effects of MDA were focused on reduction rates in the prevalence and intensity of disease [5,12-17] and have not investigated as to what extent the reduction rate changes depending on the baseline value. It therefore is critical to compare changes in the prevalence and intensity of Schistosoma infection between high- and low-prevalence areas to determine the adequate frequency of MDA; however, there have been no studies in this regard. To fill this knowledge gap, this study aimed to compare the changes in the prevalence and intensity of Schistosoma haematobium in 2 districts of the White Nile State, Sudan.

\section{MATERIALS AND METHODS}

\section{Study area}

The White Nile State is located along the White Nile River in the southeastern part of Sudan. A number of water canals connecting with the river have been developed. The Al Jabalain and El Salam districts of the White Nile State are composed of 6 and 5 Administrative Units, respectively (Fig. 1). An Admin-

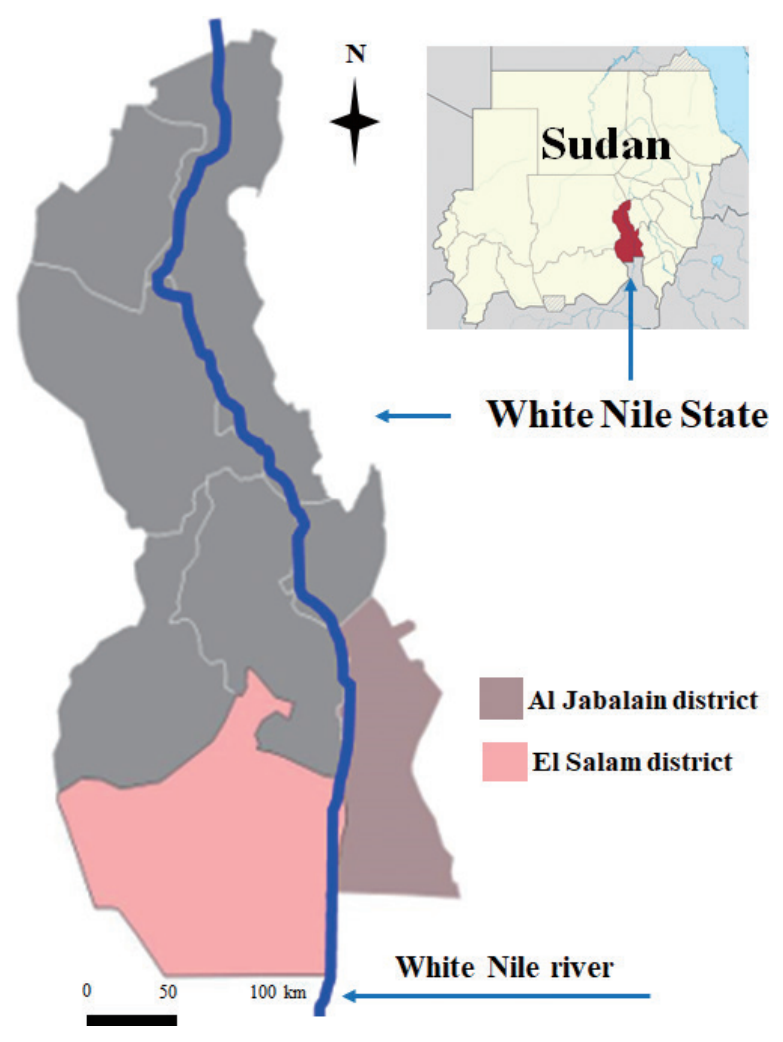

Fig. 1. Location of Al Jabalain and El Salam districts in the White Nile State, Sudan. istrative Unit is the lowest level of the local administrative organization in Sudan and has been used as a basic structure for implementing MDA.

\section{Study design and sampling}

This was a repeated cross-sectional study. Two-stage cluster sampling was used. In 2013, 6 primary schools were randomly selected among 169 and 76 schools from Al Jabalain and El Salam, respectively. Of the target schools, 2,783 and 3,197 students were sampled for baseline and follow-up surveys, respectively. The baseline survey was conducted in June 2013, the MDA intervention was carried out in July 2013, and the follow-up survey was conducted in March 2014 (Fig. 2).

In each target school, all students in 1st, 3rd, and 5th grade were sampled. For the baseline survey, 2,784 students-1,632 from Al Jabalain and 1,152 from El Salam-were sampled. For the follow-up survey, 3,197 students-1,672 from Al Jabalain and 1,525 from El Salam-were sampled (Fig. 3). The total number of participated students were different depending on the time of the survey. The increase in the number of students at the follow-up survey was due to the rise in the enrollment and attendance rates in 2014 compared to 2013.

Their urine samples were assessed for testing $S$. haematobium infection using centrifugation methods. After the baseline survey, MDA was conducted targeting schools within Administrative Units with a prevalence of $\geq 1 \%$ according to the Sudanese government MDA policy. Accordingly, MDA was implemented in all Administrative Units targeting SACs in the 2 districts because all Administrative Units had a schistosomiasis prevalence of $\geq 1 \%$. The number of subjects for this study was estimated conveniently [18], not relying on the formula for sample size calculation mainly because the main purpose of the data collection was to assess progress and provide a report to the funder (Korea International Cooperation Agency [KOICA]). We thus had to maximize the number of samples within the given budget to assess any changes at the school level, as possible as we could.

\section{Intervention}

From 2009 until 2011, KOICA implemented an integrated schistosomiasis control project in the $\mathrm{Al}$ Jabalain district (1st phase of the KOICA project) [5]. The 2nd phase started in 2012, extending the target areas to the El Salam district. This study was conducted during the 2nd phase. The low baseline prevalence and intensity in $\mathrm{Al} \mathrm{Jabalain} \mathrm{compared} \mathrm{to} \mathrm{El} \mathrm{Salam}$ was believed to be partially due to the repeated MDA interven- 


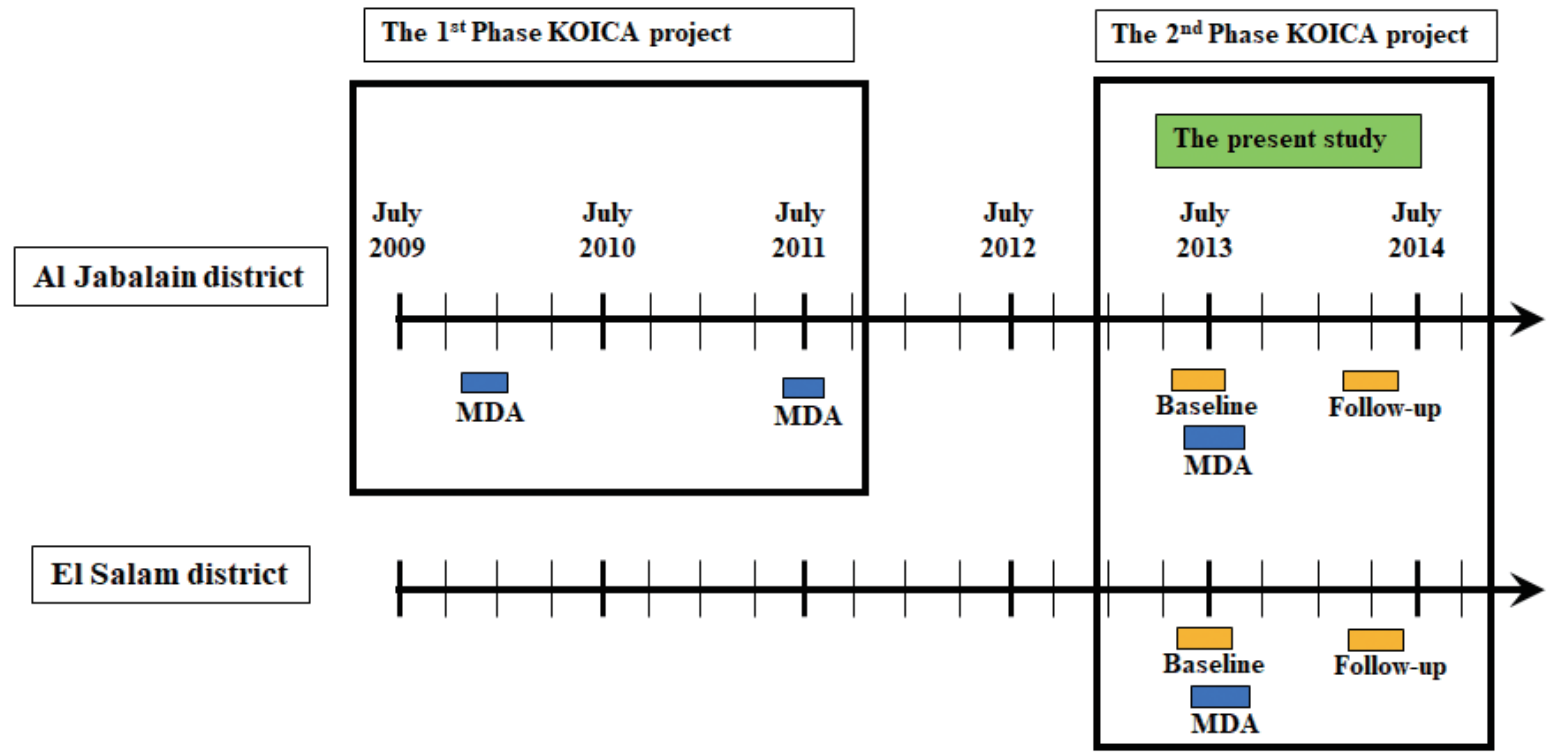

Fig. 2. Schematic illustration of the study design and mass drug administration (MDA) with praziquantel to school-aged children in Al Jabalain and El Salam districts in the White Nile State of Sudan.

\section{The $2^{\text {nd }}$ Phase KOICA Project in White Nile State of Sudan}

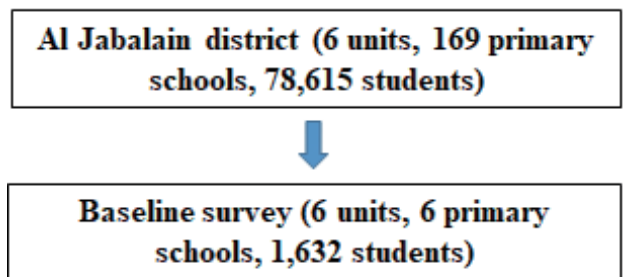

schools, 1,632 students)
El Salam district (5 units, 76 primary schools, 15,934 students)

Baseline survey (5 units, 6 primary schools, 1,152 students)

$\longleftarrow$ Urine collection and examination

MDA with praziquantel (6 units, 169 primary schools, 72,435 students)

MDA with praziquantel (5 units, 76 primary schools, 13,119 students)

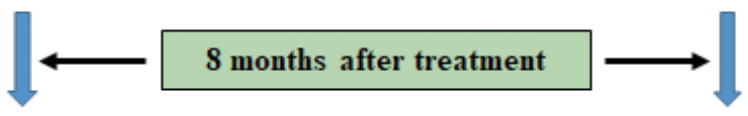

Follow-up survey ( 6 units, 6 primary schools, 1,672 students)
Follow-up survey (5 units, 6 primary schools, 1,525 students)

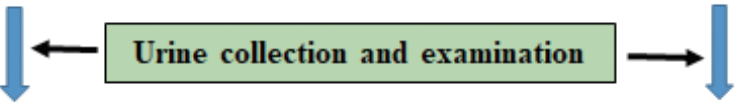

Data analysis between baseline survey and follow-up survey

- Prevalence of S. haematobium infection and its reduction pattern

- Infection intensity of S. haematobium infection and its reduction pattern

Fig. 3. Flowchart of participation and study methods. This project was carried out in Al Jabalain and El Salam districts, White Nile State, Sudan 2013-2014. 
tions during the 1st phase. In contrast, El Salam had never received any MDA intervention from 2009 up until the baseline data collection in 2012. During the 2nd phase of the project, SACs in both districts received MDA intervention. Details of the study design and MDA during the 1st and 2nd phases of the KOICA project are illustrated in Fig. 3. Details of the MDA intervention during the 2nd phase are described in Table 1.

The chemotherapy strategy followed the schistosomiasis control protocol of the Sudanese government, which was a modified treatment strategy recommended by the World Health Organization (WHO) [19]. Parasitology experts controlled all drug treatments. The drugs were delivered through the school-based system by health workers. All health workers were White Nile State Ministry of Health officials and were educated on drug treatment and schistosomiasis control projects by parasitology experts before the study. For the MDA of praziquantel (Distocide ${ }^{\circledR}$, GMC Co. Ltd., Khartoum, Sudan), health workers went to schools in the morning and administered praziquantel to the SACs directly. In this study, we administered praziquantel to all primary school students regardless of the baseline prevalence of schistosomiasis in 2013, according to the schistosomiasis control protocol of the Sudanese government. The WHO dose pole was used to determine the dosage of praziquantel $(40 \mathrm{mg} / \mathrm{kg}$ ) for SACs [19]. The drug coverage rates among SACs were $92.1 \%$ in Al Jabalain and 82.3\% in El Salam.

\section{Ethics statement}

The study protocol was reviewed and approved by the Insti- tutional Review Board (IRB) of the Korea Association of Health Promotion (KAHP) (IRB approval no. 12-C-01) and received further ethical clearance from the Federal Ministry of Health of Sudan. Before the commencement of the study at each school, health workers explained the study objectives to the schoolmaster and teachers and obtained verbal informed consent from them prior to the recruitment of children. After that, verbal informed assent was obtained from each student with the presence of the schoolteachers. The requirement for written informed consent from each student was waived by the IRB. Data were collected from the primary-school students according to the IRB-approved framework.

\section{Urine sample collection and examination}

The prevalence and intensity of $S$. haematobium infection were assessed using urine samples collected from all students in 1st, 3rd, and 5th grade of each school. Containers were delivered early in the morning to the schools and collected on the same day (usually around 2-4 pm before children were leaving school). The collected samples were immediately transferred to Sudan-Korea Schistosomiasis Control Center established by the KOICA project (the SUKO Center) in Kosti district, White Nile State.

After collection, parasitological examinations were performed on the same day by laboratory technicians with $>5$ years of experience in urine and stool microscopy. S. haematobium eggs were detected in the urine samples via the urine centrifugation methods $[13,20]$. Specifically, $10 \mathrm{ml}$ of urine in each tube was centrifuged at 1,500 rpm for 5 minutes at room

Table 1. Number of target primary school children treated with praziquantel for control of schistosomiasis in Al Jabalain and El Salam districts, White Nile Sate, Sudan

\begin{tabular}{lccccc}
\hline District & Administrative unit & No. of schools & No. of school children & No. of treated & Coverage rate (\%) \\
\hline Al Jabalain & Al Jabalain & 30 & 10,226 & 9,992 & 9,116 \\
& Assalaya & 29 & 10,444 & 5,043 & 87.7 \\
& Jazeera Aba & 18 & 6,474 & 3,644 & 77.9 \\
& Joda & 10 & 4,463 & 17,191 & 81.6 \\
& Kenana & 34 & 17,750 & 27,449 & 96.9 \\
& Rabak & 48 & 29,258 & 72,435 & 93.8 \\
El Salam & Subtotal & 169 & 78,615 & 4,194 & 92.1 \\
& Al Rawat & 30 & 5,321 & 2,089 & 78.8 \\
& Al Zelit & 12 & 2,602 & 3,438 & 80.3 \\
Al Naeem & 18 & 4,313 & 1,696 & 79.7 \\
& Al Kewaik & 7 & 1,892 & 1,702 & 89.6 \\
& Al Migainis & 9 & 1,806 & 13,119 & 94.2 \\
\end{tabular}


temperature, and the sediments were transferred onto 2-4 glass slides to examine the whole pellet. The slides were examined via microscopy to detect and count $S$. haematobium eggs. The intensity of $S$. haematobium infection was expressed as geometric mean intensity (GMI) of eggs per $10 \mathrm{ml}$ of urine (EP10) and classified into 2 categories: light intensity $(\mathrm{EP} 10<50)$ and heavy intensity $(\mathrm{EP} 10 \geq 50)$ [20]. For quality control, $10 \%$ of slides were randomly selected and re-examined at the end of each day by parasitology experts who were blinded to the results of the first examination. In case of disagreement, the results were discussed with the concerned technician, and the discordant slides were re-examined until an agreement was reached.

\section{Statistical analysis}

SPSS version 24.0 software (SPSS Inc., San Diego, California, USA) was used to analyze the experimental data. Due to the deviation from a normal distribution, $S$. haematobium infection intensities were calculated as GMIs of EP10. Differences in continuous variables among groups were tested using the MannWhitney test and Student's $t$-test. The reduction rate of prevalence and infection intensity was calculated by the following formula: reduction rate $=$ (prevalence or infection intensity at baseline survey-prevalence or infection intensity at follow-up survey)/prevalence or infection intensity at baseline survey $\times 100$.

\section{RESULTS}

\section{Reduction rate of prevalence after MDA intervention}

The baseline prevalence of $S$. haematobium infection was $9.1 \%$ in $\mathrm{Al}$ Jabalain and $35.2 \%$ in El Salam. The overall prevalence was $19.9 \%$ at the baseline survey in 2013 and 3.6\% at the follow-up survey in 2014, indicating an $82 \%$ reduction rate after MDA $(P<0.001 ; 95 \%$ confidence interval [CI], 82$88 \%$ ). The prevalence in $\mathrm{Al}$ Jabalain was reduced from $9.1 \%$ at

Table 2. The prevalence rate of $S$. haematobium infection of children of sentinel primary schools at Al Jabalain and El Salam district at baseline and 8 months after MDA

\begin{tabular}{|c|c|c|c|c|c|c|c|c|c|c|}
\hline \multirow[b]{2}{*}{ District } & \multirow[b]{2}{*}{ Variable } & \multicolumn{3}{|c|}{ Baseline survey } & \multicolumn{3}{|c|}{ Follow-up survey } & \multirow[b]{2}{*}{$\begin{array}{l}\text { Reduction } \\
\text { rate (\%) }\end{array}$} & \multirow[b]{2}{*}{$95 \% \mathrm{Cl}$} & \multirow[b]{2}{*}{$P$-value } \\
\hline & & No. exam & $\begin{array}{c}\text { No. } \\
\text { positive }\end{array}$ & $\%$ & No. exam & $\begin{array}{c}\text { No. } \\
\text { positive }\end{array}$ & $\%$ & & & \\
\hline \multirow[t]{8}{*}{ Al Jabalain } & Unit & & & & & & & & & \\
\hline & Al Jabalain & 291 & 80 & 27.5 & 312 & 17 & 5.4 & 80.2 & $0.09-026$ & $<0.001$ \\
\hline & Assalaya & 250 & 28 & 11.2 & 300 & 7 & 2.3 & 79.2 & $0.08-0.44$ & $<0.001$ \\
\hline & Jazeera Aba & 129 & 4 & 3.1 & 148 & 0 & 0.0 & 100 & & 0.996 \\
\hline & Joda & 300 & 3 & 1.0 & 305 & 1 & 0.3 & 67.2 & $0.03-3.15$ & 0.332 \\
\hline & Kenana & 451 & 18 & 4.0 & 300 & 2 & 0.7 & 83.3 & $0.04-0.70$ & 0.015 \\
\hline & Rabak & 211 & 16 & 7.6 & 307 & 3 & 1.0 & 87.1 & $0.04-0.42$ & $<0.001$ \\
\hline & Subtotal & 1,632 & 149 & 9.1 & 1,672 & 30 & 1.8 & 80.3 & $0.12-0.27$ & $<0.001$ \\
\hline \multirow[t]{7}{*}{ El Salam } & Unit & & & & & & & & & \\
\hline & Al Rawat & 188 & 83 & 44.1 & 301 & 25 & 8.3 & 81.2 & $0.07-0.19$ & $<0.001$ \\
\hline & Al Zelit & 291 & 131 & 45.0 & 315 & 5 & 1.6 & 96.5 & $0.01-0.05$ & $<0.001$ \\
\hline & Al Naeem & 312 & 105 & 33.7 & 300 & 31 & 10.3 & 69.3 & $0.15-0.35$ & $<0.001$ \\
\hline & Al Kewaik & 150 & 33 & 22.0 & 304 & 2 & 0.7 & 97.0 & $0.01-0.10$ & $<0.001$ \\
\hline & Al Migainis & 211 & 54 & 25.6 & 305 & 21 & 6.9 & 73.1 & $0.13-0.37$ & $<0.001$ \\
\hline & Subtotal & 1,152 & 406 & 35.2 & 1,525 & 84 & 5.5 & 84.4 & $0.08-0.14$ & $<0.001$ \\
\hline \multirow[t]{7}{*}{ Total } & Gender & & & & & & & & & \\
\hline & Boys & 2,127 & 424 & 19.9 & 2,328 & 88 & 3.8 & 81.0 & $0.12-0.20$ & $<0.001$ \\
\hline & Girls & 657 & 131 & 19.9 & 869 & 26 & 3.0 & 85.0 & $0.08-0.19$ & $<0.001$ \\
\hline & Age group & & & & & & & & & \\
\hline & $\leq 9$-year-old & 1,032 & 188 & 18.2 & 1,157 & 28 & 2.4 & 86.7 & $0.07-0.17$ & $<0.001$ \\
\hline & 10 12-year-old & 1,048 & 218 & 20.8 & 1,313 & 57 & 4.3 & 79.1 & $0.13-0.23$ & $<0.001$ \\
\hline & $\geq 13$-year-old & 704 & 149 & 21.2 & 727 & 29 & 4.0 & 81.2 & $0.10-0.23$ & $<0.001$ \\
\hline Total & & 2,784 & 555 & 19.9 & 3,197 & 114 & 3.6 & 82.1 & $0.12-0.18$ & $<0.001$ \\
\hline
\end{tabular}

*The follow-up survey was conducted at 8 months after MDA. 95\% Cl; 95\% confidence intervals.

Reduction Rate $=$ (prevalence at baseline survey-prevalence at follow-up survey)/prevalence at baseline survey $\times 100$. 
Table 3. The geometric mean intensity of $S$. haematobium-infected children of sentinel primary schools at Al Jabalain and El Salam districts at baseline and 8 months of follow-up

\begin{tabular}{|c|c|c|c|c|c|c|c|}
\hline \multirow{2}{*}{ District } & \multirow{2}{*}{ Variable } & \multicolumn{2}{|c|}{ Baseline survey } & \multicolumn{2}{|c|}{ Follow-up survey } & \multirow{2}{*}{ Reduction rate } & \multirow{2}{*}{$P$-value } \\
\hline & & No. positive & GMI & No. positive & GMI & & \\
\hline \multirow[t]{8}{*}{ Al Jabalain } & Unit & & & & & & \\
\hline & Al Jabalain & 80 & 15.8 & 17 & 8.0 & 49.4 & $<0.001$ \\
\hline & Assalaya & 28 & 10.8 & 7 & 7.8 & 27.8 & $<0.001$ \\
\hline & Jazeera Aba & 4 & 22.8 & 0 & 0 & 100 & \\
\hline & Joda & 3 & 12.6 & 1 & 2 & 84.1 & 0.31 \\
\hline & Kenana & 18 & 8.0 & 2 & 4.9 & 38.8 & 0.01 \\
\hline & Rabak & 16 & 28.1 & 3 & 5.2 & 81.5 & $<0.001$ \\
\hline & Subtotal & 149 & 14.5 & 30 & 7.1 & 51.0 & $<0.001$ \\
\hline \multirow[t]{7}{*}{ El Salam } & Unit & & & & & & \\
\hline & Al Rawat & 83 & 24.8 & 25 & 10.4 & 58.1 & $<0.001$ \\
\hline & Al Zelit & 131 & 17.4 & 5 & 3.5 & 79.9 & $<0.001$ \\
\hline & Al Naeem & 105 & 18.7 & 31 & 19.6 & -4.8 & $<0.001$ \\
\hline & Al Kewaik & 33 & 9 & 2 & 4.9 & 45.6 & $<0.001$ \\
\hline & Al Migainis & 54 & 20.8 & 21 & 7.8 & 62.5 & $<0.001$ \\
\hline & Subtotal & 406 & 18.5 & 84 & 11.2 & 39.5 & $<0.001$ \\
\hline \multirow[t]{7}{*}{ Total } & Gender & & & & & & \\
\hline & Boys & 424 & 17.8 & 88 & 12.3 & 30.9 & $<0.001$ \\
\hline & Girls & 131 & 15.8 & 26 & 4.8 & 69.6 & $<0.001$ \\
\hline & Age group & & & & & & \\
\hline & $\leq 9$-year-old & 188 & 18.3 & 28 & 9.3 & 49.2 & $<0.001$ \\
\hline & 10 12-year-old & 218 & 17.4 & 57 & 11.0 & 36.8 & $<0.001$ \\
\hline & $\geq 13$-year-old & 149 & 16.0 & 29 & 8.7 & 45.6 & $<0.001$ \\
\hline Total & & 555 & 17.3 & 114 & 9.5 & 45.1 & $<0.001$ \\
\hline
\end{tabular}

${ }^{*} \mathrm{GMl}$ (Geometric mean intensity); Number of $\mathrm{S}$. haematobium eggs per $10 \mathrm{ml}$ of urine.

Reduction rate of infection intensity $=($ GMl at baseline survey-GMl at follow-up survey $) / G M l$ at baseline survey $\times 100$.

the baseline survey to $1.8 \%$ at the follow-up survey, hence a reduction rate of $80.3 \%(P<0.001 ; 95 \% \mathrm{CI}, 73-88 \%)$. In $\mathrm{El}$ Salam, the prevalence was $35.2 \%$ at the baseline survey, which was reduced to $5.5 \%$ at the follow-up survey, thus an $84.4 \%$ reduction rate $(P<0.001 ; 95 \% \mathrm{CI}, 86-92 \%)$. No significant differences in relative reduction of prevalence rates were observed between both districts (Table 2).

\section{Reduction rate of infection intensity after MDA intervention}

Table 3 shows the intensity of $S$. haematobium infection at the baseline and follow-up surveys in Al Jabalain and El Salam. The overall GMI for both districts was 17.3 EP10 at the baseline survey and 9.5 EP10 at the follow-up survey; thus, the overall reduction in GMI was 45\%, showing a significant difference between the baseline and follow-up surveys $(P<$ 0.001). The GMI of infection in $\mathrm{Al}$ Jabalain was reduced from 14.5 EP10 at baseline to 7.1 EP10 at 8 months of follow-up, accounting for $51.0 \%$ of relative reduction $(P<0.001)$. In El Salam, the GMI of infection at baseline and at 8 months of follow-up was 18.5 and 11.2 EP10, respectively, which constituted $40 \%$ of relative reduction $(P<0.001)$.

As shown in Table 4, 7.7\% of children had a heavy intensity of infection at the baseline survey $(2.9 \%$ in $\mathrm{Al}$ Jabalain and $14.4 \%$ in El Salam). After MDA, the prevalence of heavy infection was significantly reduced in $\mathrm{Al}$ Jabalain (93.9\% reduction rate, $P<0.001)$ and El Salam $(93.2 \%$ reduction rate, $P<0.001)$ (Table 4).

\section{DISCUSSION}

Human schistosomiasis caused by S. haematobium is one of the major public health concerns in Sudan. Previous studies showed that the prevalence of $S$. haematobium infection in the White Nile State of Sudan was 28.5\% [5], 45.0\% [6], 21.9\% [21], and 35.6\% [22] in different survey areas and times. In this study, we compared changes in the prevalence and intensity of S. haematobium infection between Al Jabalain and El Salam districts of White Nile State before and after MDA inter- 
Table 4. The relative percentages of heavy and light intensities of $S$. haematobium infected children of sentinel primary schools at Al Jabalain and El Salam districts at baseline and 8 months of follow-up

\begin{tabular}{|c|c|c|c|c|c|c|c|c|c|c|}
\hline \multirow[b]{2}{*}{ District } & \multirow[b]{2}{*}{ Variable } & \multicolumn{3}{|c|}{ Baseline survey } & \multicolumn{3}{|c|}{ Follow-up survey } & \multirow[b]{2}{*}{$\begin{array}{l}\text { Reduction } \\
\text { rate (\%) }\end{array}$} & \multirow[b]{2}{*}{$95 \% \mathrm{Cl}$} & \multirow[b]{2}{*}{$P$-value } \\
\hline & & Total no. & $\begin{array}{c}\text { Light } \\
\text { infection } \\
(\%)\end{array}$ & $\begin{array}{c}\text { Heavy } \\
\text { infection (\%) }\end{array}$ & Total no. & $\begin{array}{l}\text { Light } \\
\text { infection } \\
(\%)\end{array}$ & $\begin{array}{c}\text { Heavy } \\
\text { infection (\%) }\end{array}$ & & & \\
\hline \multirow[t]{8}{*}{ Al Jabalain } & Unit & & & & & & & & & \\
\hline & Al Jabalain & 291 & 18.6 & 8.9 & 312 & 4.8 & 0.6 & 92.8 & $0.06-0.30$ & $<0.001$ \\
\hline & Assalaya & 250 & 8.8 & 2.4 & 300 & 2.0 & 0.3 & 86.1 & $0.08-0.89$ & 0.03 \\
\hline & Jazeera Aba & 129 & 0.9 & 0.9 & 148 & 0 & 0 & 100 & & \\
\hline & Joda & 300 & 0.7 & 0.3 & 305 & 0.3 & 0 & 100 & & \\
\hline & Kenana & 451 & 3.1 & 0.9 & 300 & 0.7 & 0 & 100 & & \\
\hline & Rabak & 211 & 3.4 & 4.3 & 307 & 1.0 & 0 & 100 & & \\
\hline & Subtotal & 1,632 & 6.2 & 2.9 & 1,672 & 1.6 & 0.2 & 93.9 & $0.06-0.22$ & $<0.001$ \\
\hline \multirow[t]{7}{*}{ El Salam } & Unit & & & & & & & & & \\
\hline & Al Rawat & 188 & 20.7 & 23.4 & 301 & 7.3 & 1.0 & 95.7 & $0.05-0.17$ & $<0.001$ \\
\hline & Al Zelit & 291 & 26.5 & 18.6 & 315 & 1.6 & 0 & 100 & & \\
\hline & Al Naeem & 312 & 21.2 & 12.5 & 300 & 6.3 & 4.0 & 68 & $0.31-0.69$ & $<0.001$ \\
\hline & Al Kewaik & 150 & 18.0 & 4.0 & 304 & 0.7 & 0 & 100 & & \\
\hline & Al Migainis & 211 & 14.7 & 10.9 & 305 & 6.9 & 0 & 100 & & \\
\hline & Subtotal & 1,152 & 20.8 & 14.4 & 1,525 & 4.5 & 1.0 & 93.2 & $0.10-0.18$ & $<0.001$ \\
\hline \multirow[t]{7}{*}{ Total } & Gender & & & & & & & & & \\
\hline & Boys & 2,127 & 12.1 & 7.9 & 2,328 & 3.0 & 0.7 & 90.6 & $0.13-0.23$ & $<0.001$ \\
\hline & Girls & 657 & 12.6 & 7.3 & 869 & 2.9 & 0.1 & 98.4 & $0.02-0.08$ & $<0.001$ \\
\hline & Age group & & & & & & & & & \\
\hline & $\leq 9$-year-old & 1,032 & 10.8 & 7.4 & 1,157 & 2.2 & 0.3 & 96.5 & $0.04-0.12$ & $<0.001$ \\
\hline & 10 12-year-old & 1,048 & 13.0 & 7.8 & 1,313 & 3.5 & 0.8 & 89.3 & $0.14-0.29$ & $<0.001$ \\
\hline & $\geq 13$-year-old & 704 & 13.2 & 8.0 & 727 & 3.4 & 0.6 & 93.1 & $0.07-0.23$ & $<0.001$ \\
\hline Total & & 2,748 & 12.2 & 7.7 & 3,197 & 3.0 & 0.6 & 92.7 & $0.11-0.18$ & $<0.001$ \\
\hline
\end{tabular}

*Light infection intensity (<50 EP10) and heavy infection intensity ( $\times 50 \mathrm{EP} 10)$ of S. haematobium infections.

Reduction rate $=(\%$ of heavy infection intensity at baseline survey- $\%$ of heavy infection intensity at follow-up survey $) / \%$ of heavy infection intensity at baseline survey $\times 100$.

vention. Prior to the baseline survey of this study, SACs in Al Jabalain had received MDA interventions twice during the 4 years of the 1st phase of the KOICA project, while those in El Salam had not. In 2013, the baseline prevalence of S. haematobium infection was 35.2\% in El Salam, which was similar to that in previous studies in the White Nile State $[5,6,22]$, while the 2013 baseline prevalence in $\mathrm{Al}$ Jabalain was 9.1\% due to previous MDA intervention within 4 years [5]. In this study, the reduction in the prevalence of infection after MDA was $80.3 \%$ in $\mathrm{Al}$ Jabalain and $84.4 \%$ in El Salam. Although both districts had different MDA histories, the chemotherapeutic effect of a single praziquantel MDA was both quite satisfactory based on the observed reduction rates of prevalence in both districts. Such reduction in the prevalence rate after MDA represents the proportion of cure among the infected population, and similar findings were observed across all sex and age categories. The reduction rate of prevalence after a single dose of praziquantel was similar to that of previous studies of SACs in Sudan and Burkina Faso $[14,15]$.

Infection intensity-which is quantified by egg counts (GMI of EP10 in this study)-is an important index of schistosomiasis epidemiology. Egg counts can estimate the burden of infecting worms and represent the degree of morbidity by eggs in the bladder [23]. In this study, we used urine sedimentation method to detect and quantify the $S$. haematobium eggs. That is why urine filtration system is blocked easily due to many foreign substances in the urine collected at the survey sites due to the fallen bladder cells, crystals and so on [24]. Also, urine sedimentation method is higher detectability even if low number of parasites and economic than urine filtration method [20]. Our results showed that the reduction rates of infection intensity after MDA were $51.0 \%$ in $\mathrm{Al}$ Jabalain and 39.5\% in $\mathrm{El}$ Salam. Unlike the results for reduction rates of prevalence, this study suggests relatively less reduction in infection intensity 
observed among the populations with heavier infection burdens. El Salam demonstrated a higher prevalence rate of urogenital schistosomiasis and higher EP10 counts than Al Jabalain. A single MDA produced a similar efficacy of reduction in the prevalence rate (i.e., cure rate) in El Salam, but a lower efficacy of worm reduction in the human body; this suggests that most of the children with a light burden of infection were cured by MDA and the proportion was similar in both districts, but heavy-burden cases remained uncured with lowered EP10 counts after MDA. Our findings indicate that a single MDA of a $40 \mathrm{mg} / \mathrm{kg}$ dose is less effective to cure a heavy burden of schistosomiasis and that repeated MDAs are recommended for control programs.

When we broke down the prevalence rates and egg counts by administrative unit in the districts, we found wide variations in findings in both localities. Even in Al Jabalain, there were administrative units with a low reduction rate of prevalence, such as the Joda and Al Naeem Administrative Units. A possible explanation might be an ongoing refugee immigration from South Sudan. Immigrants may influence the prevalence of schistosomiasis, so precautionary measures should be taken during refugee immigration and settlement. In interpreting the effects of MDA between the 2 districts, we also had to consider reinfection. We examined the urine microscopy results at 8 months after MDA, which was long enough to evaluate reinfection after MDA. A single $40 \mathrm{mg} / \mathrm{kg}$ dose of praziquantel is known to produce a $73.6 \%$ cure rate and a $94.7 \%$ egg reduction rate for $S$. haematobium infection [25]. The reduction in prevalence rate in our study was $>80 \%$, which is similar to the known cure rate $[14,15]$. However, the reduction in intensity rate (39.5\% in El Salam and 51.0\% in Al Jabalain) was much lower than the known 90\% egg reduction rate. The egg passers after the MDA included treatment failure and reinfection cases without clear identification. In heavily endemic areas, children are more rapidly and heavily reinfected after chemotherapy because they are more exposed. The reinfection force is determined by the egg seeding amount within the local area [25]. In this study, the follow-up survey was conducted 8 months after the MDA-after the hot and humid rainy season and long summer vacation had passed. Thus, SACs might have come into contact with water (e.g., during swimming) more frequently, resulting in reinfection. This study also confirmed that the reduction in infection intensity was much less in Sudan than as expected. Therefore, repeated MDAs may diminish the reinfection force by reducing the egg seeding population and the total amount of egg production in a community. Once repeated MDAs start to effectively keep the egg seeding amount under the reinfection threshold, schistosomiasis may finally be eliminated.

This study has a few limitations. Although the follow-up data at 8 months after the MDA included both treatment failure and reinfection cases after medication, it was impossible to differentiate their impacts. The other limitation was the influence of immigrants from South Sudan who were included in the study population. This aspect warrants further study.

In conclusion, we compared the effects of MDA with praziquantel in regions that are geographically adjacent but profoundly different in terms of the baseline infection status of urogenital schistosomiasis. This study showed that praziquantel administration was equally effective in reducing the prevalence of $S$. haematobium infection regardless of the baseline prevalence levels in endemic areas; however, one MDA with a single dose of praziquantel was not sufficient to reduce a heavy intensity of infection. Thus, this study provides real site information about the reduction patterns of prevalence and infection intensity after praziquantel treatment in endemic regions with different baseline prevalence levels. Our findings clearly point to the importance of repeated MDA interventions in endemic areas, which will eventually contribute to schistosomiasis elimination.

\section{ACKNOWLEDGMENTS}

This study was funded by the Korea International Cooperation Agency (KOICA). The views, opinions, assumptions, or any other information set out in this article should not be attributed to KOICA or any person connected with them. This work was partly supported by research fund of Chungnam National University.

We appreciate all the staffs involved in "The Project for Combating Schistosomiasis in Sudan," carried by Korea Association of Health Promotion (KAHP) and the Ministry of Health $(\mathrm{MoH})$ of the White Nile State, Sudan. We are indebted to several colleagues at Ministry of Health of Sudan and Embassy of the Republic of Korea in Sudan. Also, we thank all primary school children and teachers of $\mathrm{Al} \mathrm{Jabalain} \mathrm{and} \mathrm{El}$ Salam loclaities of White Nile State, Sudan. This project was supported by the Korea International Cooperation Agency (KOICA project no. P2015-00146-2) and implemented by the Korea Association of Health Promotion. This work was partly 
supported by research fund of Chungnam National University.

\section{CONFLICT OF INTEREST}

The authors declare that they have no competing interests.

\section{REFERENCES}

1. Gryseels B. Schistosomiasis. Infect Dis Clin North Am 2012; 26: 383-397.

2. Hotez PJ, Kamath A. Neglected tropical diseases in sub-Saharan Africa: review of their prevalence, distribution, and disease burden. PLoS Negl Trop Dis 2009; 3: e412.

3. GBD and HALE Collaborators. Global, regional, and national disability-adjusted life-years (DALYs) for 359 diseases and injuries and healthy life expectancy (HALE) for 195 countries and territories, 1990-2017: a systematic analysis for the Global Burden of Disease Study 2017. Lancet 2018; 392: 1859-1922.

4. World Health Organization. Fact Sheet on Schistosomiasis [Internet]; Available from: http://www.who.int/mediacentre/factsheets/fs115/en/.

5. Lee YH, Jeong HG, Kong WH, Lee SH, Cho HI, Nam HS, Ismail HA, Alla GN, Oh CH, Hong ST. Reduction of urogenital schistosomiasis with an integrated control project in Sudan. PLoS Negl Trop Dis 2015; 9: e3423.

6. Ismail HA, Hong ST, Babiker AT, Hassan RM, Sulaiman MA, Jeong HG, Kong WH, Lee SH, Cho HI, Nam HS, Oh Ch, Lee YH. Prevalence, risk factors, and clinical manifestations of schistosomiasis among school children in the White Nile River basin, Sudan. Parasit Vectors 2014; 7: 478.

7. Abou-Zeid AH, Abkar TA, Mohamed RO. Schistosomiasis infection among primary school students in a war zone, Southern Kordofan State, Sudan: a cross-sectional study. BMC Public Health 2013; 13: 643.

8. Hajissa K, Muhajir AEMA, Eshag HA, Alfadel A, Nahied E, Dahab R, Ali SM, Mohammed M, Gaafar M, Mohamed Z. Prevalence of schistosomiasis and associated risk factors among school children in Um-Asher Area, Khartoum, Sudan. BMC Res Notes 2018; 11: 779 .

9. Cha SM, Elhag MS, Lee YH, Cho DS, Ismail HA, Hong ST. Epidemiological findings and policy implications from the nationwide schistosomiasis and intestinal helminthiasis survey in Sudan. Parasit Vectors 2019; 12: 429.

10. Zhang Y, MacArthur C, Mubila L, Baker S. Control of neglected tropical diseases needs a long-term commitment. BMC Med 2010; 8: 67.

11. World Health Organization. Helminth Control in School Age Children: A Guide for Managers of Control Programmes. 2nd ed. Geneva, Switzerland. World Health Organization. 2011.

12. Atalabi TE, Adubi TO. The epidemiology and chemotherapeutic approaches to the control of urinary schistosomiasis in school- age children (SAC): a systematic review. BMC Infect Dis 2019; 19: 73.

13. Atalabi TE, Adoh SD, Eze KM. The current epidemiological status of urogenital schistosomiasis among primary school pupils in Katsina State, Nigeria: An imperative for a scale up of water and sanitation initiative and mass administration of medicines with Praziquantel. PLoS Negl Trop Dis 2018; 12: e0006636.

14. Ahmed AM, Abbas H, Mansour FA, Gasim GI, Adam I. Schistosoma haematobium infections among school children in central Sudan one year after treatment with praziquantel. Parasit Vectors 2012; 5: 108.

15. Touré S, Zhang Y, Bosqué-Oliva E, Ky C, Ouedraogo A, Koukounari A, Gabrielli AF, Bertrand S, Webster JP, Fenwick A. Two-year impact of single praziquantel treatment on infection in the national control programme on schistosomiasis in Burkina Faso. Bull World Health Organ 2008; 86: 780-787.

16. King CH, Kittur N, Binder S, Campbell CH, N'Goran EK, Meite A, Utzinger J, Olsen A, Magnussen P, Kinung'hi S, Fenwick A, Phillips AE, Gazzinelli-Guimaraes PH, Dhanani N, Ferro J, Karanja DMS, Mwinzi PNM, Montgomery SP, Wiegand RE, Secor WE, Hamidou AA, Garba A, Colley DG Impact of different mass drug administration strategies for gaining and sustaining control of Schistosoma mansoni and Schistosoma haematobium infection in Africa. Am J Trop Med Hyg 2020; 103 (suppl): 14-23.

17. Dejon-Agobé JC, Edoa JR, Honkpehedji YJ, Zinsou JF, Adégbitè BR, Ngwese MM, Mangaboula A, Lell B, Grobusch MP, Mordmüller B, Adegnika AA. Schistosoma haematobium infection morbidity, praziquantel effectiveness and reinfection rate among children and young adults in Gabon. Parasit Vectors 2019; 12: 577.

18. Hayes RJ, Bennett S. Simple sample size calculation for clusterrandomized trials. Int J Epidemiol 1999; 28: 319-326.

19. World Health Organization. Prevention and Control of Schistosomiasis and Soil-Transmitted Helminthiaisis: WHO Tech Report Series No.912. Geneva, Switzeland. World Health Organization. 2002.

20. World Health Organization. Basic laboratory methods in Medical Parasitology. Geneva, Switzeland. World Health Organization. 1991, pp 33-35.

21. Ahmed ES, Daffalla A, Christensen NO, Madsen H. Patterns of infection and transmission of human schistosomiasis mansoni and schistosomiasis haematobium in White Nile Province, Sudan. Ann Trop Med Parasitol 1996: 90: 173-180.

22. Lee YH, Lee JS, Jeoung HG, Kwon IS, Mohamed AAWS, Hong ST. Epidemiological survey on schistosomiasis and intestinal helminthiasis among village residents of the rural river basin area in White Nile State, Sudan. Korean J Parasitol 2019; 57: 135-144.

23. Andrade G, Bertsch DJ, Gazzinelli A, King CH. Decline in infection-related morbidities following drug-mediated reductions in the intensity of Schistosoma infection: a systematic review and meta-analysis. PLoS Negl Trop Dis 2017; 11: e0005372.

24. Kim MJ, Ryu K, Jin Y, Lee YH, Jeoung HG, Saeed AA, Kim SH, Hong ST. Significance of echogenic snow sign as an ultrasonog- 
raphy finding for diagnosis of urogenital schistosomiasis. Am J Trop Med Hyg 2016; 95: 842-848.

25. Zwang J, Olliaro P. Efficacy and safety of praziquantel $40 \mathrm{mg} / \mathrm{kg}$ in preschool-aged and school-aged children: a meta-analysis. Parasit Vectors 2017; 10: 47. 